\title{
Procesos de agenda y políticas de desarrollo urbano: centrando la cuestión
}

\section{Governance and urban development policies: theory and practice}

\author{
Moneyba González Medina (coord.) \\ Universidad Autónoma de Madrid \\ moneyba.gonzalez@uam.es
}

\section{NOTA BIOGRÁFICA}

Doctora en Ciencia Política y de la Administración (Mención Europea) por la Universidad de Santiago de Compostela (2009). Es profesora e investigadora del Departamento de Ciencia Política y Relaciones Internacionales de la Universidad Autónoma de Madrid. Su investigación se enmarca en el análisis de las políticas públicas y la modernización de las administraciones, con especial atención al ámbito urbano.

\section{María Ángeles Huete García (coord.) Universidad Pablo de Olavide de Sevilla mahuegar@upo.es}

\section{NOTA BIOGRÁFICA}

Es profesora e investigadora del Departamento de Sociología de la Universidad Pablo de Olavide, de Sevilla. Responsable del Grupo de Investigación en Gobernanza Urbana y Metropolitana (GUM-Z) (Sej-606). Es doctora en Sociología (Mención Europea) por la Universidad Pablo de Olavide (2008). Su investigación se centra en el análisis de políticas públicas y gobernanza, con especial atención al ámbito urbano.

\section{RESUMEN}

Presentación del número monográfico Gobernanza y políticas de desarrollo urbano: teoría y práctica.

\section{PALABRAS CLAVE}

Gobernanza urbana; desarrollo urbano sostenible integrado; agendas urbanas.

\section{ABSTRACT}

Preface of the monographic issue Governance and policies of urban development: theory and practice.

\section{KEYWORDS}

Urban Governance, integrated sustainable urban development; urban agendas.

\section{SUMARIO}

La promoción de una Política urbana nacional. Una nueva ola de reformas metropolitanas. Activación de los procesos de «agenda urbana». El enfoque de Desarrollo Urbano Sostenible Integrado (DUSI). I. Retos y nuevas formas de gobernanza. II. El enfoque de Desarrollo Urbano Sostenible Integrado. Referencias bibliográficas. III. La aplicación del enfoque DUSI en España: la iniciativa URBANA. 
Desde la década de los noventa, la cuestión urbana ha recabado una creciente atención. Las estadísticas $^{1}$ arrojan datos que, año tras año, desvelan la relevancia de las ciudades en términos demográficos, económicos, sociales y culturales. Son lugares donde se concentran los problemas y las oportunidades (CE, 2011), que requieren políticas públicas cada vez más complejas tanto en su dimensión sustantiva como procedimental (González, 2011; Huete et al., 2016). Las ciudades se convierten, de este modo, en laboratorios de innovación democrática y gobernanza, en espacios de experimentación donde se afrontan nuevos (y viejos) retos de desarrollo urbano de manera sostenible e integrada. Sin embargo, lo urbano ha comenzado a tener una mayor presencia en el ámbito político solo en los últimos años. La puesta en marcha de programas que promueven la construcción de una Política urbana nacional (UN-Habitat y OECD, 2018), el impulso de reformas institucionales que recuperan la cuestión metropolitana o la reciente activación de las denominadas «agendas urbanas» son algunos ejemplos.

El interés por profundizar en estas cuestiones motivó la constitución de un grupo de trabajo sobre "Políticas de desarrollo urbano en Europa: Teoría y Práctica" en el XIII Congreso de la Asociación Española de Ciencia Política y de la Administración (AECPA), celebrado en Santiago de Compostela (20-22 de septiembre de 2017). El grupo, coordinado por Moneyba González Medina (Universidad Autónoma de Madrid) y María Ángeles Huete García (Universidad Pablo de Olavide de Sevilla), contó con la colaboración de Carmen Navarro (Universidad Autónoma de Madrid) y Rafael Merinero Rodríguez (Universidad Pablo de Olavide de Sevilla) como comentaristas. Su objetivo era analizar nuevas formas de gobernanza y políticas de desarrollo urbano, con especial atención a los procesos de «agenda urbana» y al «enfoque DUSI». El número de propuestas, la variedad de territorios representados (Andalucía, Asturias, Cataluña, Madrid y Valencia) y el diverso background de los participantes (Arquitectura y Urbanismo, Ciencia Política, Derecho², Ecología, Geografía, Informática o Sociología) no solo era un reflejo del extendido interés por lo urbano en distintas disciplinas, sino sobre todo de la complejidad que le es inherente. La presente publicación recoge algunos de estos trabajos, que pueden organizarse en tres grandes bloques: I. Retos y nuevas formas de gobernanza; II. El enfoque de Desarrollo Urbano Sostenible Integrado; y III. La aplicación del enfoque DUSI en España: iniciativa URBANA.

A continuación, se describirán de forma muy sucinta los procesos que sirven de marco a estos trabajos, para finalizar dando algunas pinceladas sobre las principales ideas y enfoques empleados por los distintos autores y autoras que participan en esta publicación. Finalmente, destacamos la oportunidad de este número de la Revista Gestión y Análisis de Políticas Públicas, que coincide, además, con la inminente aprobación de la Agenda Urbana Española ${ }^{3}$.

\section{LA PROMOCIÓN DE UNA POLÍTICA URBANA NACIONAL}

La cuestión de una Política de y para las ciudades ha entrado en la agenda política mundial, europea y nacional de muchos países. El hecho es llamativo si se tiene en cuenta que son pocos los países que han contado tradicionalmente con una Política urbana explícita, entendida como un conjunto de medidas de gobierno dirigidas a las ciudades, adoptadas en diferentes niveles administrativos (europeo, nacional, regional o local) (Van der Berg et al., 2007). Se trata de un fenómeno nuevo que se caracteriza, además, por manifestarse con independencia del grado de desarrollo del territorio donde se inscribe. La literatura que analiza la cuestión es reciente y cada vez más numerosa (Allulli y Tortorella, 2013; González y Fedeli, 2015; Friendly, 2016; González et al., 2017), al igual que los documentos oficiales que proponen a los gobiernos ir en esta dirección ${ }^{4}$ (UN-Habitat, 2014; 2015; OECD, 2017; UN-Habitat y OECD, 2018).

\footnotetext{
1 OECD Metropolitan Database: https://stats.oecd.org/Index.aspx?Datasetcode=CITIES

2 En el grupo de trabajo hubo contribuciones de URBAN RED, una Red Temática sobre Desarrollo Urbano Sostenible, coordinada por María Rosario Alonso Ibáñez (Universidad de Oviedo), que tuvo su origen en un proyecto enmarcado en la Convocatoria 2015 de Acciones de dinamización «Redes de Excelencia», del Programa Estatal de Investigación Científica y Técnica de Excelencia, Subprograma Estatal de Generación de Conocimiento, en el marco del Plan Estatal de Investigación Científica y Técnica y de Innovación 2013-2016 (Proyecto: DER2015-71345-REDT). Más información: https://www.unioviedo.es/Desarrollosostenible/urban-red/index.php.

3 Se trata de un momentum de centralidad política de lo urbano sin precedentes en España, que ha ido vertebrándose a caballo entre el enfoque DUSI impulsado por la Unión Europea y los ODS de Naciones Unidas. En concreto, de los 17 Objetivos de Desarrollo Sostenible recogidos en la Agenda 2030 (2015), el ODS 11: Ciudades y Comunidades Sostenibles, es específico de las ciudades.

4 Tras HABITAT III, se puso en marcha The National Urban Policy Programme (NUPP), una iniciativa conjunta de UN Habitat, OECD y Cities Alliance, dirigida a trabajar de manera conjunta para dar apoyo al desarrollo e implementación de las Políticas Urbanas Nacionales a nivel global. Más información: http://www.oecd.org/gov/national-urban-policies.htm.
} 


\section{UNA NUEVA OLA DE REFORMAS METROPOLITANAS}

Junto a ello, se observa un retorno de la cuestión metropolitana, especialmente en Europa. Por ejemplo, en 2014, países como Francia o Italia impulsaron reformas institucionales dirigidas a formalizar y «anclar» el hecho metropolitano en sus sistemas políticos. Ello dio lugar a muchos debates acerca de en qué escuela de pensamiento inscribir el proyecto o cuál era el mejor diseño institucional. Sin embargo, ciertamente, gran parte de estas reformas obedecieron más a razones de racionalización presupuestaria que a un auténtico deseo de formalizar jurídica y políticamente esta «categoría estadística» o «funcional», lo que ha sido interpretado por algunos autores como una manifestación de posicionamientos neoliberales por parte de la UE (Lang y Török, 2017).

\section{ACTIVACIÓN DE LOS PROCESOS DE «AGENDA URBANA»}

Pero quizás lo más llamativo haya sido la activación de procesos para la elaboración de «agendas urbanas» a escala global, europea, nacional y subnacional. En cuestión de pocos años, se han aprobado la Nueva Agenda Urbana (2016), la Agenda Urbana para la Unión Europea (2016), la Agenda Urbana del Eixo Atlántico (2017) -cuya originalidad reside en su carácter transfronterizo-, la Agenda Urbana de Andalucía (2018) y previsiblemente, a finales de este año, la Agenda Urbana Española. Además, este mismo fenómeno se observa en países tan distintos como Angola, Canadá, India o Italia (Gelli, 2016).

\section{FIGURA 1. DESARROLLO DE LOS PROCESOS DE AGENDA URBANA}

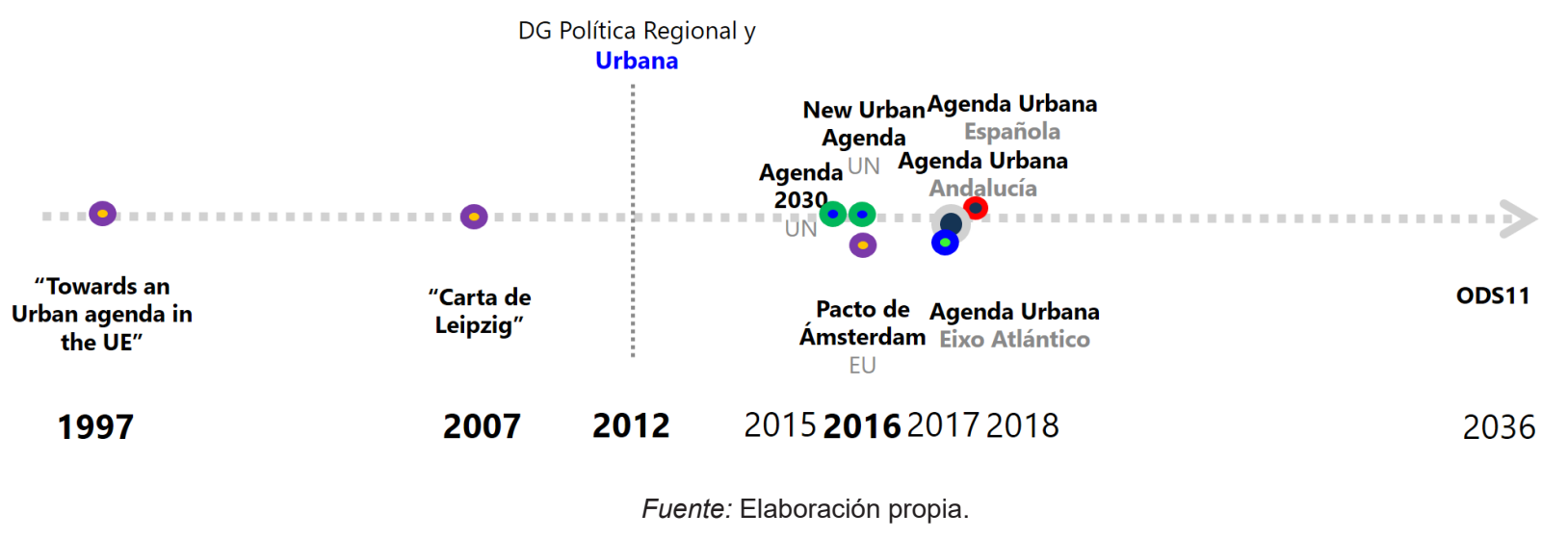

\section{EL ENFOQUE DE DESARROLLO URBANO SOSTENIBLE INTEGRADO (DUSI)}

Por otra parte, bajo la rúbrica de la innovación social y el desarrollo sostenible, han ido apareciendo nuevas formas de organización y de colaboración entre actores institucionales y no institucionales, orientadas a encontrar soluciones a problemas o demandas urbanas. Algunas de estas iniciativas son el resultado de procesos de autoorganización colectiva «desde abajo» (por ejemplo, la PAH), enmarcadas, muchas veces en el ideario del «derecho a la ciudad». Otras, por el contrario, han sido impulsadas «desde arriba», con el objetivo de proveer marcos operativos para el diseño e implementación de proyectos de desarrollo urbano diferenciados de las tradicionales «maneras de hacer» de la administración (por ejemplo, el enfoque DUSI) o para la normalización del uso de las TICs en el ámbito urbano (por ejemplo, el modelo Smart City).

En concreto, el enfoque de Desarrollo Urbano Sostenible Integrado (DUSI), promovido por la UE, ha tenido una gran influencia en España. En palabras de Del Castillo y Haarich, «el principio de desarrollo urbano integrado ${ }^{5}$ se refiere más a cuestiones relacionadas con la planificación y la gestión del proceso de desarroIlo, mientras que el carácter sostenible está más ligado a los objetivos, temas y áreas de intervención para el 2014-2020

5 Este principio está recogido en el artículo 8 del reglamento del FEDER 2007-2013 y en el artículo 7 del reglamento del FEDER 
GAPP. Nueva Época - N.ํ 20, noviembre 2018 - ISSN: 1989-8991 - DOI: 10.24965/gapp.v0i20.10594 - [Págs. 6-13] Número monográfico - Gobernanza y políticas de desarrollo urbano: teoría y práctica

Procesos de agenda y políticas de desarrollo urbano: centrando la cuestión Moneyba González Medina / María Ángeles Huete García (coords.)

desarrollo urbano. En la práctica, ambos conceptos van de la mano» (2013: 17). EI DUSI (CE, 2014a; 2014b; 2015) es el resultado de más de dos décadas de implementación de proyectos integrados de desarrollo urbano, que arrancan con los Urban Pilot Project (1989-1999) y la Iniciativa comunitaria URBAN (1994-2006) (De Gregorio, 2017). Es, fundamentalmente, un método de trabajo caracterizado por su carácter holístico, multi-actor (o multinivel), participativo y estratégico, que permite alinear los proyectos de desarrollo urbano impulsados en las distintas ciudades europeas con las prioridades y objetivos de la Política de cohesión de la UE, de acuerdo con un modelo europeo del desarrollo urbano (CE, 2011). En otras palabras, el DUSI es el core o la raison d'être de la «Política urbana» de la UE.

\section{MAPA 1. IMPLEMENTACIÓN DEL DUSI 2014-2020}

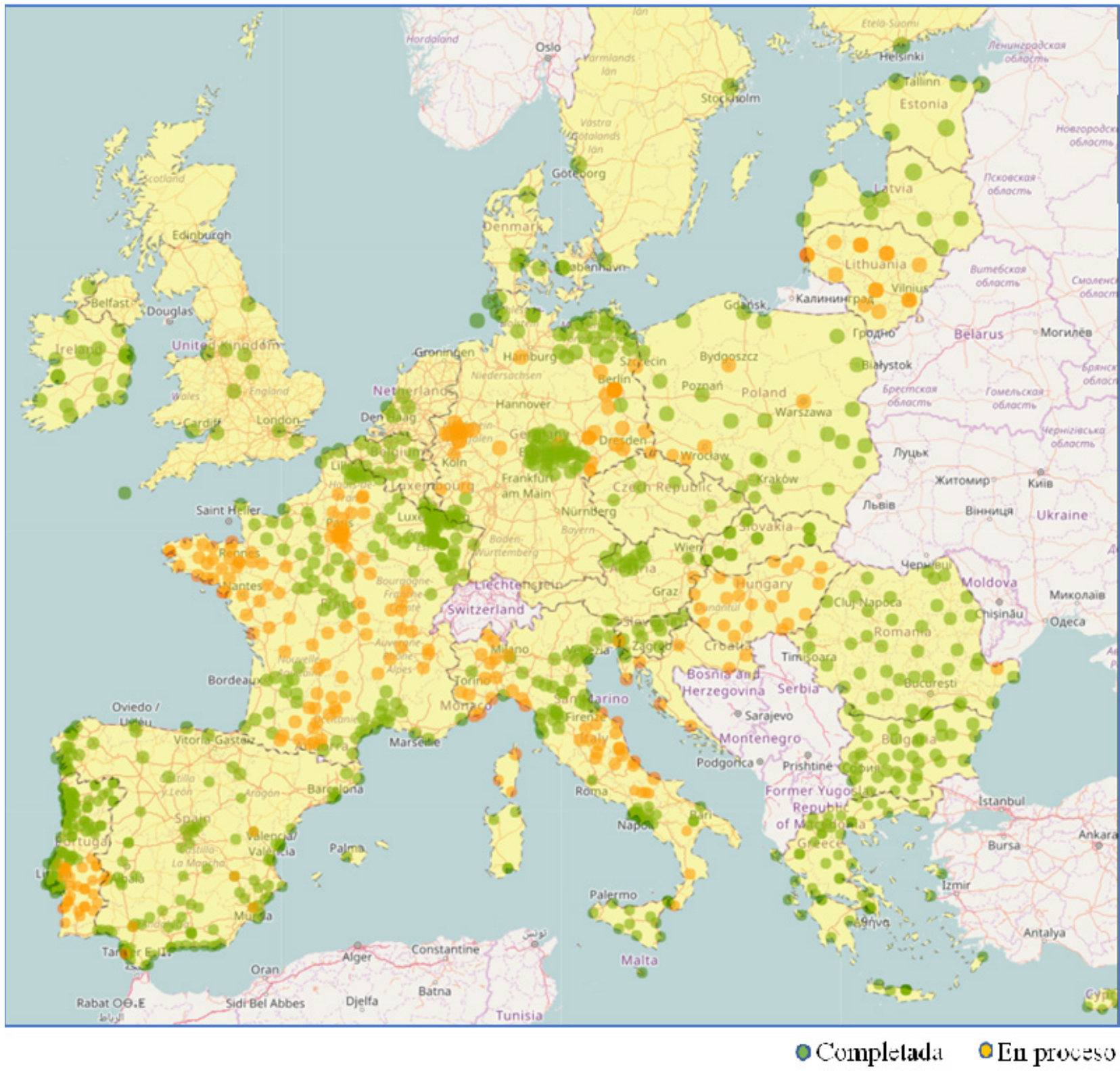

Fuente: STRAT-Board ${ }^{6}$

6 STRAT-BOARD es una herramienta de mapeo interactivo que ofrece una panorámica de la implementación del DUSI, con cargo a los Fondos EIE 2014-2020, en los 28 Estados Miembros. Datos a 19 de octubre de 2018. Más información: https://urban.jrc.ec.europa. eu/strat-board/\#/home. 
EI DUSI ha tenido una gran influencia en España, donde se ha verificado un efecto de europeización urbana down-load, entendido como un proceso de acomodación (domesticación) de las reglas formales e informales promovidas UE en el ámbito del desarrollo urbano en un Estado Miembro (González et al., 2017). Se encuentran evidencias de este proceso, por ejemplo, en el desarrollo de la capacidad institucional de muchas administraciones locales, cuyo personal reconoce que los proyectos de desarrollo urbano financiados con Fondos Europeos exigen formas de trabajo diferenciadas de la práctica administrativa ordinaria (Busetti et al., 2018). España, se caracteriza, además, por ser el único Estado Miembro que, durante cuatro periodos de programación consecutivos, ha impulsado de manera continuada programas de desarrollo urbano integrado (URBANA 2007-2013 y EDUSI 2014-2020) en línea con los principios promovidos por la UE (De Gregorio, 2018). Todos estos procesos han preparado el terreno para el lanzamiento de la Agenda Urbana Española (Acuerdo de Asociación de España, 2014: 222).

FIGURA 2. EVOLUCIÓN DEL ENFOQUE DE DUSI EN ESPAÑA

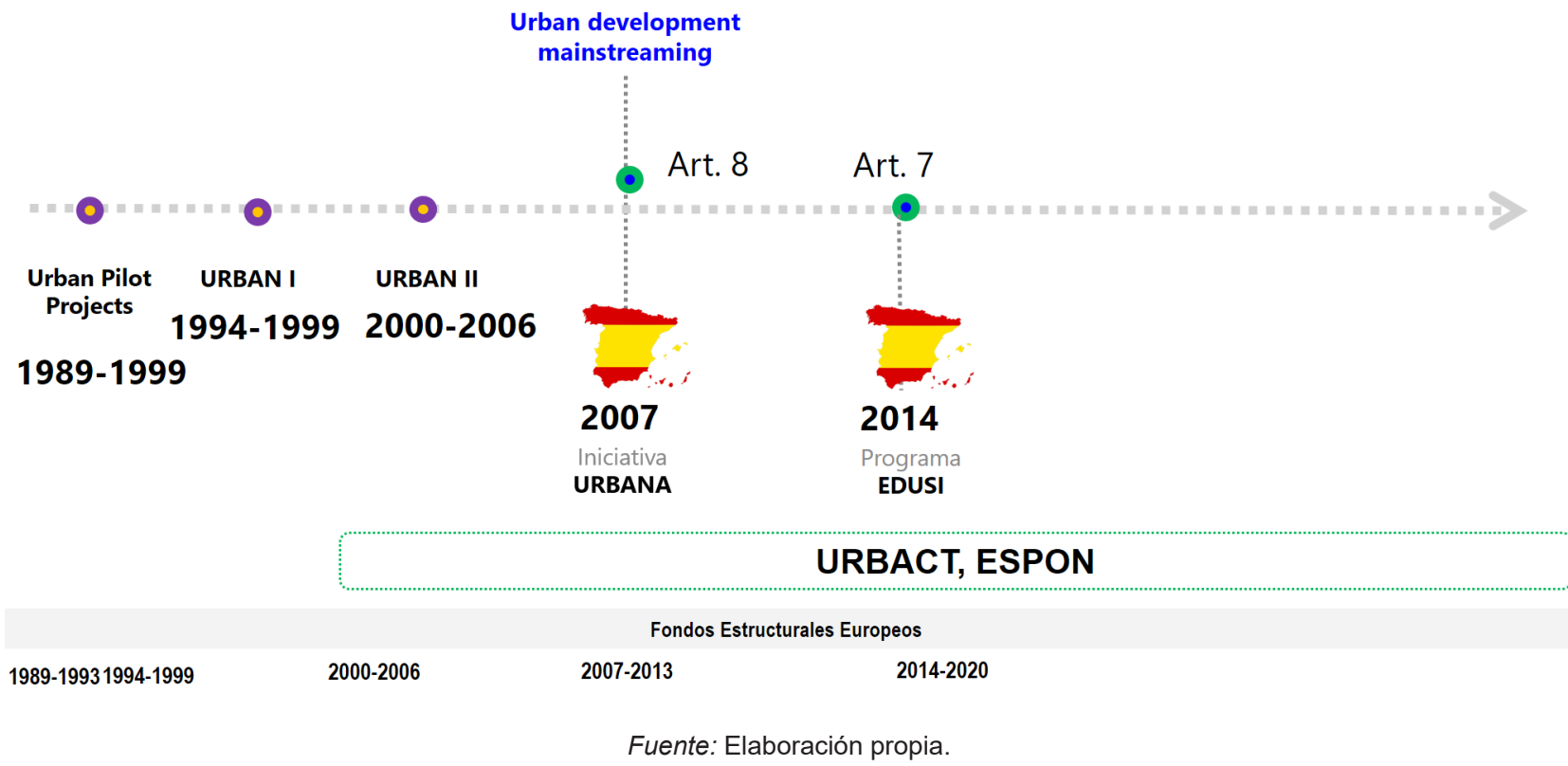

En el presente número de la revista GAPP se abordan todos estos procesos desde distintas perspectivas. Los artículos no tienen un único estilo, ni representan una misma escuela. Combinan un enfoque teórico y práctico, abarcan distintas escalas territoriales (grandes ciudades, ciudades medias y barrios) y han sido elaborados por autores y autoras con distintos background académicos y metodologías de investigación. A continuación, se describirán las principales ideas y enfoques desarrollados por cada uno de ellos.

\section{RETOS Y NUEVAS FORMAS DE GOBERNANZA}

En El nuevo municipalismo: derecho a la ciudad y comunes urbanos, Ricard Gomà, Ismael Blanco y Joan Subirats abordan los retos clave de empoderamiento de las ciudades en redes horizontales de gobernanza. Analizan las distintas etapas del municipalismo en España, planteando la posible configuración de un nuevo municipalismo definido por dos dimensiones: una agenda de políticas urbanas comprometidas con el derecho a la ciudad y una agenda de prácticas urbanas vinculadas a la construcción del común. La Obra Social de la PAH es el caso escogido para evidenciar la articulación de ambos aspectos con relación al derecho a la vivienda.

Adela Romero se aproxima a la cuestión de las Smart Cities desde un punto de vista teórico, en un trabajo titulado El paradigma de las Smart Cities en el marco de la gobernanza urbana. Su objetivo es desarrollar este paradigma en el marco de la gobernanza, conectándolo con los retos de la participación, la ética pública, la sostenibilidad, la transparencia, la eficacia y eficiencia, y la rendición de cuentas. 
Por su parte, Joaquín Farinós y Pilar Lloret analizan el caso de «Decidim València» en La dimensión participativa en el diseño de políticas públicas urbanas. El caso valenciano. Se trata de una iniciativa de consulta ciudadana de inversión por barrios desarrollada por el Ayuntamiento de Valencia. Los autores se detienen en todas las fases del proceso de consulta ciudadana y hacen un balance de los problemas de implementación a los que se ha enfrentado y de sus impactos. Lo hacen, concretamente, desde el marco teórico de la Do-ocrazy, en el que los ciudadanos cambian su rol tradicional en la producción y gestión de la ciudad, pasando de la protesta a la propuesta.

Los siguientes trabajos se enmarcan en el ámbito de la gobernanza metropolitana, si bien abordan la cuestión de dos maneras muy diferenciadas. En Políticas europeas y gobernanza metropolitana: una asignatura pendiente, Mariona Tomàs realiza un ejercicio de encuadre teórico de los modelos de gobernanza metropolitana en Europa a partir de cuatro variables: la fragmentación del territorio, las competencias de naturaleza metropolitana, el tipo de financiación y el tipo de representación. Igualmente llama la atención sobre el hecho de que, si bien las políticas europeas están progresivamente ampliando y reforzando su dimensión urbana y metropolitana, el papel de las autoridades metropolitanas existentes sigue siendo secundario. Por su parte, Pedro Limón presenta la cuestión metropolitana desde una perspectiva muy distinta. En concreto plantea una crítica al enfoque DUSI y a lo que denomina la «producción de globalidad» a través de mega-eventos en grandes ciudades. En su trabajo Gobernanza metropolitana y producción de globalidad en Madrid y Barcelona se detiene en dos ejemplos de gentrificación consolidados en Madrid y Barcelona: los barrios de Villaverde Alto y Trinitat Nova, respectivamente.

\section{EL ENFOQUE DE DESARROLLO URBANO SOSTENIBLE INTEGRADO}

El segundo bloque de trabajos se centra en el enfoque DUSI. En su artículo Evolución de los parámetros del enfoque integrado en las políticas urbanas en los barrios vulnerables en España, Ángela Matesanz y Agustín Hernández analizan la evolución de los elementos ligados al enfoque integrado en el discurso jurídico-administrativo de las políticas dirigidas a «regenerar» la ciudad consolidada en España, especialmente en barrios vulnerables. Desde una perspectiva más aplicada, Blanca del Espino y Daniel Navas describen cómo han empleado el Reference Framework for Sustainable Cities ${ }^{7}$ (RFSC), una herramienta web destinada a facilitar la aplicación del DUSI, en el II Plan Estratégico de Lucena, lanzado en 2015. Los detalles sobre esta experiencia se explican en Planeamiento estratégico local y evaluación del DesarroIlo Urbano Sostenible Integrado en ciudades medias.

\section{LA APLICACIÓN DEL ENFOQUE DUSI EN ESPAÑA: LA INICIATIVA URBANA}

Finalmente, el tercer bloque de trabajos se centra en el análisis del impacto de la iniciativa URBANA, promovida por el Gobierno de España, en el periodo 2007-2013, en el contexto de la programación del FEDER. En su trabajo, Diseño e implementación de políticas públicas de regeneración urbana en Alicante, Cádiz, Córdoba, y Coslada, Rocío Muñoz y María Ángeles Huete García tratan de identificar los factores que explican el desajuste entre el diseño y la implementación en cuatro iniciativas URBANA. Entre ellos, destaca la falta de estabilidad política, lo que avalaría la necesidad de adoptar un mayor consenso para elaborar un proyecto común de territorio.

Por último, en el artículo titulado La influencia de la iniciativa URBANA 2007-2013 en el desarrollo de la capacidad institucional de la administración municipal, Valle Casado, María Ángeles Huete, Rafael Merinero y Moneyba González Medina realizan un ejercicio de análisis de la capacidad institucional de las administraciones locales españolas como resultado de los programas cofinanciados con Fondos Europeos, prestando especial atención a la relación que existe entre su nivel de desarrollo y otros elementos del contexto local como pueden ser la experiencia previa acumulada en programas similares y las características del modelo de gestión del programa.

En definitiva, todos estos trabajos comparten su interés por lo urbano. En el contexto de los procesos de agenda y los «nuevos» enfoques de política de desarrollo urbano, sus contribuciones constituyen un es-

\footnotetext{
7 Más información en: http://rfsc.eu/.
} 
GAPP. Nueva Época - N. 20, noviembre 2018 - ISSN: 1989-8991 - DOI: 10.24965/gapp.v0i20.10594 - [Págs. 6-13] Número monográfico - Gobernanza y políticas de desarrollo urbano: teoría y práctica

Procesos de agenda y políticas de desarrollo urbano: centrando la cuestión Moneyba González Medina / María Ángeles Huete García (coords.)

fuerzo por centrar la cuestión y visibilizarla en la agenda de investigación en materia de políticas públicas en España. El tema y el momento lo merecen porque cada vez hay más las evidencias de que «El tiempo nuevo que empezamos a vivir es un tiempo urbano, la era de las ciudades...» (Gomà et al., 2018).

\section{REFERENCIAS BIBLIOGRÁFICAS}

ALLULLI, M. y TORTORELLA, W. (2013): "Cities in search of Policy", en Métropoles [Online], núm. 12, Online since 31 May 2013, connection on 30 September 2016. URL: http://metropoles.revues.org/4654.

BUSETTI, S.; GONZÁLEZ MEDINA, M.; HUETE GARCÍA, M. A. y MERINERO ROdRÍGUEZ, R. (2018): "Los mecanismos causales de la participación social: una aplicación a la regeneración urbana”, en Revista Española de Investigaciones Sociológicas, núm. 164, págs. 77-96. DOI: http://dx.doi.org/10.5477/cis/reis.164.77.

COMISIÓN EUROPEA (2011): Cities of tomorrow. Challenges, visions, ways forward. Bruselas. DOI: http://dx.doi. org/10.2776/41803.

COMISIÓN EUROPEA (2014a): Integrated Sustainable Urban Development. Factsheet. Bruselas. DOI: http://dx.doi. org/10.2776/47320.

COMISIÓN EUROPEA (2014b): Communication from the Commission to the European Parliament, the Council, the European Economic and Social Committee and the Committee of the Regions: The urban dimension of EU policies - Key features for an EU Urban Agenda. COM (2014) 490 Final, Bruselas.

COMISIÓN EUROPEA (2015): Orientaciones para los Estados miembros Desarrollo sostenible integrado en el medio urbano Reglamento del FEDER (artículo 7). Bruselas.

DEL CASTILLO, J. y HAARICH, S. N. (2013): Desarrollo Urbano Sostenible cofinanciado por el FEDER en España 2014-2020: Directrices Estratégicas y Prioridades de Inversión. Final Report for the DG Regio.

DE GREGORIO HURTADO, S. (2017): "La política urbana de la UE en España: de URBAN a las EDUSI", en TRIA - Territorio della Ricerca su Insediamenti e Ambiente, núm. 18, págs. 47-74. DOI: http://dx.doi.org/10.6092/2281$4574 / 5309$.

DE GREGORIO HURTADO, S. (2018): "The EU urban policy in the period 2007-13: lessons from the Spanish experience", Regional Studies, Regional Science, 5:1, 212-230, DOI: https://doi.org/10.1080/21681376.2018.148 0903.

FRIENDLY, A. (2016): "National Urban Policy: A Roadmap for Canadian Cities", en IMFG Perspectives, núm. 14/2016, Institute on Municipal Finance and Governance, Toronto.

GELLI, F. (2016): "The changing geography of national urban agendas and urban policies. The cases of EU, China, Brazil, USA, Angola in perspective", comunicación presentada en EURA Conference, Turín, Italia, 16-18 de Junio.

GONZÁLEZ MEDINA, M. (2011): "Elementos de una política urbana europea. Experiencias de gestión del desarrollo urbano en Alemania y España", en Revista Española de Ciencia Política, núm. 27, págs. 45-67.

GONZÁLEZ MEDINA, M. y FEDELI, V. (2015). "Exploring European Urban policy: Towards an EU-national urban agenda?”, en Gestión y Análisis de Políticas Públicas, núm. 14, julio-diciembre, págs. 8-22. DOI: http://dx.doi. org/10.24965/gapp.v0i14.10287.

GONZÁLEZ MEDINA, M.; DE GREGORIO HURTADO, S.; HUETE GARCÍA, M. A. y CARPENTER, J. (2017): "Europeización y Política urbana de la UE: Impacto en las agendas urbanas nacionales de España, Francia, Italia y Reino Unido", comunicación presentada en el XIII Congreso AECPA, Santiago de Compostela, 20-22 de Septiembre.

HUETE GARCÍA, M. A.; MERINERO RODRÍGUEZ, R. y MUÑOZ, R. (2016): "Urban regeneration policy from the Model of Integrated Urban Development in the European Union: an analytical approach based on the study of Spanish cities", en Local Government Studies, vol. 42, núm. 2, págs. 267-286.

LANG, T. y TÖRÖK, I. (2017): "Metropolitan region policies in the European Union: following national, European or neoliberal agendas?", en International Planning Studies, vol. 22, núm. 1, págs. 1-13. DOI: https://doi.org/10.1080/1 3563475.2017 .1310652

OECD (2017): National Urban Policy in OECD Countries. París: OECD Publishing. DOI: http://dx.doi. org/10.1787/9789264271906-en.

OECD (2018): OECD Regions and Cities at a Glance 2018. París: OECD Publishing. DOI: https://doi.org/10.1787/reg_ cit glance-2018-3-en.

UN-Habitat (2014): New Generation of National Urban Policies. Nairobi: United Nations Human Settlements Programme.

UN-Habitat (2015): Issue Paper on Urban Governance. HABITAT III ISSUE PAPERS, núm. 6. Nairobi: United Nations Human Settlements Programme.

UN-Habitat (2016a): Nueva Agenda Urbana, aprobada durante Hábitat III Conferencia de las Naciones Unidas sobre Vivienda y Desarrollo Urbano Sostenible, Quito, Ecuador, 17-20 de octubre.

UN-Habitat (2016b). World Cities Report. Urbanization and Development. Emerging Futures. Nairobi: United Nations Human Settlements Programme. 
GAPP. Nueva Época - N.o 20, noviembre 2018 - ISSN: 1989-8991 - DOI: 10.24965/gapp.v0i20.10594 - [Págs. 6-13] Número monográfico - Gobernanza y políticas de desarrollo urbano: teoría y práctica

Procesos de agenda y políticas de desarrollo urbano: centrando la cuestión Moneyba González Medina / María Ángeles Huete García (coords.)

UN-Habitat y OECD (2018): Global State of National Urban Policy. Nairobi: United Nations Human Settlements Programme. DOI: http://dx.doi.org/10.1787/9789264290747-en.

VAN DER BERG, L.; BRAUN, E. y VAN DER MEER, J. (2007): National Policy Responses to Urban Challenges in Europe. Hampshire: Ashgate Publishing Limited.

VV.AA (2018), "El poder de las ciudades", en Vanguardia [Dossier], núm. 67 (enero). Barcelona; La Vanguardia Ediciones, S. L. 